\title{
Additive Effects of Spironolactone and Candesartan on Cardiac Sympathetic Nerve Activity and Left Ventricular Remodeling in Patients with Congestive Heart Failure
}

\author{
Shu Kasama ${ }^{1,2}$, Takuji Toyama ${ }^{1}$, Hiroyuki Sumino ${ }^{2}$, Naoya Matsumoto ${ }^{3}$, Yuichi Sato ${ }^{3}$, Hisao Kumakura ${ }^{2}$, \\ Yoshiaki Takayama ${ }^{2}$, Shuichi Ichikawa ${ }^{2}$, Tadashi Suzuki ${ }^{1}$, and Masahiko Kurabayashi ${ }^{1}$ \\ ${ }^{1}$ Department of Cardiovascular Medicine, Gunma University School of Medicine, Maebashi, Japan; ${ }^{2}$ Department of Internal Medicine, \\ Cardiovascular Hospital of Central Japan, Gunma, Japan; and ${ }^{3}$ Department of Cardiology, Nihon University School of Medicine, \\ Tokyo, Japan
}

\begin{abstract}
The activation of the renin-angiotensin-aldosterone system prevents the uptake of norepinephrine in the myocardium. However, the additive effects of combined spironolactone and candesartan on cardiac sympathetic nerve activity (CSNA) have not been determined. We investigated the effects of the angiotensin-receptor blocker candesartan alone and in combination with spironolactone on CSNA in patients with congestive heart failure (CHF). Methods: Fifty patients with CHF (left ventricular ejection fraction [LVEF] $<45 \%$ ) were randomly assigned to candesartan plus spironolactone (group $\mathrm{A} ; n=25$ ) or to candesartan alone (group $\mathrm{B} ; n=25$ ). All patients were also treated with a loop diuretic. The delayed percent denervation, delayed heart-tomediastinum count $(\mathrm{H} / \mathrm{M})$ ratio, and washout rate $(\mathrm{WR})$ were determined from ${ }^{123}$-metaiodobenzylguanidine (MIBG) scintigraphy, and plasma brain natriuretic peptide (BNP) concentration was measured before and 6 mo after treatment. The LV enddiastolic volume (LVEDV), LV end-systolic volume (LVESV), and LVEF were also determined by echocardiography. Results: After $6 \mathrm{mo}$, all of these parameters were improved in both groups. However, the degree of change in the percent denervation was $-14 \pm 12$ in group $A$ and $-7 \pm 10$ in group $B(P<0.05)$; the change in the $\mathrm{H} / \mathrm{M}$ ratio was $0.19 \pm 0.18$ in group $A$ and $0.08 \pm$ 0.14 in group $B(P<0.05)$, the change in WR was $-12 \% \pm$ $8 \%$ in group $A$ and $-5 \% \pm 13 \%$ in group $B(P<0.05)$, and the change in plasma BNP was $-100 \pm 83 \mathrm{pg} / \mathrm{mL}$ in group $A$ and $-43 \pm 97 \mathrm{pg} / \mathrm{mL}$ in group $B(P<0.05)$. The degree of change in LVEDV, LVESV, and LVEF in group $A$ tended to be better than that in group $B$, but these changes were not statistically significant. Moreover, there were significant correlations between changes in the 123 I-MIBG scintigraphic findings and changes in the LVEDV (\% denervation, $r=0.692, P<0.001 ; \mathrm{H} / \mathrm{M}$ ratio, $r=-0.437, P<0.05$; and WR, $r=0.505, P<0.01)$ or the LVESV (\% denervation, $r=0.663, P<0.001 ; \mathrm{H} / \mathrm{M}$ ratio, $r=-0.438, P<$ 0.05 ; and WR, $r=0.532, P<0.01$ ) in group $\mathrm{A}$. In contrast, there
\end{abstract}

Received Jul. 18, 2007; revision accepted Sep. 6, 2007.

For correspondence or reprints contact: Shu Kasama, MD, Department of Cardiovascular Medicine, Gunma University School of Medicine, 3-39-15,

Showa-machi, Maebashi, Gunma 371-0034, Japan.

E-mail: s-kasama@bay.wind.ne.jp

COPYRIGHT @ 2007 by the Society of Nuclear Medicine, Inc. was no relationship between these parameters in group B. Conclusion: These findings indicate that the combination of spironolactone and candesartan may be more beneficial for CSNA and LV performance than candesartan alone in patients with CHF.

Key Words: angiotensin; aldosterone; sympathetic nervous system

J Nucl Med 2007; 48:1993-2000

DOI: 10.2967/jnumed.107.045427

O ne of the most important physiologic systems involved in the development and progression of congestive heart failure (CHF) is the renin-angiotensin-aldosterone system (RAAS). Aldosterone independently promotes potassium and magnesium loss, sodium retention, ventricular arrhythmias in humans, and, as shown in animal models, cardiac and vascular fibrosis as well as vascular necrosis $(1,2)$. These effects are mediated by the action of aldosterone on mineralocorticoid receptors in the kidney, brain, heart, and vasculature (3). The angiotensin-receptor blocker (ARB) candesartan targets the renin-angiotensin elements of RAAS, and this agent improves the quality of life and decreases the mortality and morbidity in patients with CHF (4). However, despite ARB treatment, there is still production of aldosterone, a phenomenon known as aldosterone escape or aldosterone breakthrough (1).

Cardiac imaging with ${ }^{123}$ I-metaiodobenzylguanidine (MIBG), an analog of norepinephrine, is a useful tool for detecting abnormalities of the myocardial adrenergic nervous system in patients with $\mathrm{CHF}(5,6)$. Furthermore, cardiac sympathetic nerve activity (CSNA) evaluated by ${ }^{123}$ I-MIBG scintigraphy has useful prognostic value in patients with CHF $(6,7)$. Many studies have suggested that treatment of heart failure in patients with $\mathrm{CHF}$ can improve CSNA, as evaluated by cardiac ${ }^{123}$ I-MIBG scintigraphy ( 8 19). However, the additive effects of combined spironolactone 
and candesartan on CSNA have not been determined. Accordingly, this study was performed to determine whether the combination of spironolactone and candesartan can improve CSNA compared with candesartan alone in patients with CHF.

\section{MATERIALS AND METHODS}

\section{Study Population}

The study included 50 patients with heart failure (left ventricular ejection fraction $[\mathrm{LVEF}]<45 \%$ and New York Heart Association [NYHA] functional class III or IV), who were admitted to our institution and whose symptoms had improved to NYHA functional class II or III within 1 mo with standard therapy for CHF. Patients with severe heart failure requiring mechanical support (intraaortic balloon pumping, LV assist device, cardiac resynchronization therapy, or implantable cardioverter-defibrillator) or patients requiring heart transplantation were excluded. Patients with unstable angina pectoris, renal failure, liver dysfunction, cardiogenic shock, or hypotension defined as a systolic blood pressure $<90 \mathrm{~mm} \mathrm{Hg}$ were also excluded. The study was approved by the ethics review board of Cardiovascular Hospital of Central Japan, and written informed consent was obtained from all patients.

\section{Study Protocol}

In the acute phase, all patients were treated with standard therapy for heart failure, including intravenously administered vasodilators and diuretics. All patients also received oral angiotensin-converting enzyme (ACE) inhibitor and loop diuretic (furosemide). When the patient was compensated with oral medications alone for at least $2 \mathrm{wk}, 50$ patients were randomly assigned (double-blind, 1:1 ratio) to either candesartan plus spironolactone (group $\mathrm{A} ; n=25$ ) or candesartan alone (group B; $n=25$ ) instead of an ACE inhibitor. Candesartan was started at a dose of $2-4 \mathrm{mg} / \mathrm{d}$ in all patients, and the dose was gradually increased to a maximum of $8-12 \mathrm{mg} / \mathrm{d}$. In group A, spironolactone was also started at a dose of $25 \mathrm{mg} / \mathrm{d}$ at the same time. In this study, the $\beta$-blocker carvedilol was not started for all CHF patients. In both group A and group B, carvediol was added to the study medications in the patients who needed to stabilize the symptoms of heart failure. This agent was finally administered to 36 of 50 patients (group A, $n=17$ [68\%]; group B, $n=19[76 \%]$ ). Carvedilol was started at a dose of $1.25-$ $2.5 \mathrm{mg} / \mathrm{d}$ in these patients, and the dose was gradually increased to a maximum of $10-20 \mathrm{mg} / \mathrm{d}$. Examinations were performed before and after candesartan and spironolactone treatment for a mean of 6 mo in all CHF patients.

\section{Echocardiography}

Echocardiography was performed using standard methods in a blinded manner: 2 experienced, independent echocardiographers who had no knowledge about the study performed all measurements. The LV end-diastolic volume (LVEDV), LV end-systolic volume (LVESV), and LVEF were calculated using the modified method of Simpson (20).

\section{3|-MIBG Imaging}

The ${ }^{123}$ I-MIBG imaging method has been described previously (21-23). ${ }^{123}$ I-MIBG was obtained from a commercial source (FUJIFILM RI Pharma Co. Ltd.). Patients were injected ${ }^{123}{ }^{1-}$ MIBG (111 MBq) intravenously while in the supine position. At $15 \mathrm{~min}$ and at $4 \mathrm{~h}$ after the injection, static data were acquired in the anterior view with a single-head $\gamma$-camera (Millennium MPR;
GE Healthcare) equipped with a low-energy, general-purpose, parallel-hole collimator. Static images on a $128 \times 128$ matrix were collected for 5 min with a $20 \%$ window centered on $159 \mathrm{keV}$, corresponding to the ${ }^{123}$ I photopeak. After the static planar images were acquired, SPECT images were obtained. The camera was rotated over $180^{\circ}$ from the $45^{\circ}$ right anterior oblique position to the $45^{\circ}$ left posterior oblique position in 32 views with an acquisition time of $40 \mathrm{~s}$ per view. Scans were acquired in a $64 \times$ 64 matrix by a filtered backprojection method for reconstruction.

The heart-to-mediastinum count $(\mathrm{H} / \mathrm{M})$ ratio was determined from the anterior planar delayed ${ }^{123}$ I-MIBG image. The washout rate (WR) was calculated using the following formula: $\{([\mathrm{H}]-$ $[\mathrm{M}])$ early $-([\mathrm{H}]-[\mathrm{M}])$ delayed $\} /([\mathrm{H}]-[\mathrm{M}])$ early $\times 100(\%)$, where $[\mathrm{H}]=$ mean counts per pixel in the left ventricle and $[\mathrm{M}]=$ mean counts per pixel in the upper mediastinum. In this study, time decay was not corrected for the calculation of WR.

The delayed myocardial SPECT images of each patient were divided into 17 segments as recommended by American Heart Association (24). Regional tracer uptake was assessed semiquantitatively using a 5-point scoring system $(0=$ normal uptake, $1=$ mildly reduced uptake, $2=$ moderately reduced uptake, $3=$ significantly reduced uptake, and $4=$ no uptake). The total defect score (TDS) was calculated as the sum of all defect scores. The TDS was converted to a percentage of the total denervated myocardium ( $\%$ denervation). The $\%$ denervation was calculated using the following formula: $(\mathrm{TDS} / 68$ [maximum score $=4 \times 17$ ] $\times 100$ ).

\section{Plasma Brain Natriuretic Peptide (BNP) Concentrations}

Blood samples were collected into tubes containing ethylenediaminetetraacetic acid after the patient had rested in a supine position for at least $30 \mathrm{~min}$. Plasma was separated by centrifugation and frozen at $-84^{\circ} \mathrm{C}$. Then the plasma concentration of BNP was measured with a specific immunoradiometric assay for human BNP using a commercially available kit (Shionogi) as previously reported (14-16,25).

\section{Data Analysis and Statistics}

Statistical analysis was performed by using SPSS 12.0 for Windows (SPSS Inc.). The numeric results are expressed as the mean $\pm \mathrm{SD}$. Comparison of baseline categoric data between the 2 groups was done by the 1 -sided $\chi^{2}$ contingency table method, and differences between continuous variables were evaluated using the unpaired $t$ test. Changes in NYHA functional class were assessed using the Wilcoxon matched pairs signed rank test. In patients who underwent repeated assessment, changes from baseline were evaluated within each treatment group using a paired $t$ test and between the 2 groups using 2-way ANOVA. Linear regression analysis was used to determine the relationship between continuous variables. In all analyses, $P<0.05$ was considered statistically significant.

\section{RESULTS}

\section{Clinical Characteristics}

There were no significant differences in hemodynamic characteristics or cardiac medications between the 2 groups on entry into the study. The cause of heart failure was ischemic heart disease (IHD) $(n=23)$, idiopathic dilated cardiomyopathy (DCM) $(n=18)$, or other diseases $(n=9$ (mitral regurgitation, $n=4$; aortic regurgitation, $n=3$; hypertensive heart disease, $n=2$ ). At baseline, the $\%$ denervation, H/M ratio, WR, LVEDV, LVESV, LVEF, 
TABLE 1

Clinical Characteristics of Patients

\begin{tabular}{|c|c|c|c|}
\hline Characteristic & $\begin{array}{c}\text { Group A* } \\
(n=25)\end{array}$ & $\begin{array}{c}\text { Group B* } \\
(n=25)\end{array}$ & $P$ \\
\hline Age (y) & $69 \pm 12$ & $67 \pm 9$ & 0.508 \\
\hline Sex & & & 0.765 \\
\hline Male & $16(64)$ & $17(68)$ & \\
\hline Female & $9(36)$ & $8(32)$ & \\
\hline NYHA functional class & & & 0.771 \\
\hline II & $9(36)$ & $10(40)$ & \\
\hline III & $16(64)$ & $15(60)$ & \\
\hline \multicolumn{4}{|l|}{ Cause of $\mathrm{CHF}$} \\
\hline IHD & $10(40)$ & $13(52)$ & 0.395 \\
\hline DCM & $10(40)$ & $8(32)$ & 0.556 \\
\hline Others & $5(20)$ & $4(16)$ & 0.713 \\
\hline \multicolumn{4}{|l|}{ 123|-MIBG } \\
\hline$\%$ denervation & $54 \pm 10$ & $55 \pm 14$ & 0.773 \\
\hline $\mathrm{H} / \mathrm{M}$ ratio & $1.63 \pm 0.23$ & $1.68 \pm 0.18$ & 0.396 \\
\hline WR & $47 \pm 10$ & $46 \pm 10$ & 0.725 \\
\hline \multicolumn{4}{|l|}{ Echocardiography } \\
\hline LVEDV (mL) & $192 \pm 32$ & $187 \pm 39$ & 0.622 \\
\hline LVESV (mL) & $131 \pm 30$ & $130 \pm 32$ & 0.910 \\
\hline LVEF (\%) & $32 \pm 6$ & $31 \pm 6$ & 0.558 \\
\hline Plasma BNP (pg/mL) & $249 \pm 133$ & $243 \pm 119$ & 0.867 \\
\hline \multicolumn{4}{|l|}{ Medical treatment } \\
\hline Candesartan & $25(100)$ & $25(100)$ & 1.000 \\
\hline Spironolactone & $25(100)$ & - & - \\
\hline Carvedilol & $17(68)$ & $19(76)$ & 0.529 \\
\hline Furosemide & $25(100)$ & $25(100)$ & 1.000 \\
\hline
\end{tabular}

NYHA functional class, and plasma BNP concentrations were similar in both groups (Table 1).

The mean maintenance dose of candesartan was $10.0 \pm$ $1.9 \mathrm{mg} / \mathrm{d}$ in group A versus $10.2 \pm 1.8 \mathrm{mg} / \mathrm{d}$ in group B $(P=$ not significant $[\mathrm{NS}])$. The mean dose of carvedilol was $13 \pm 6 \mathrm{mg} / \mathrm{d}$ in group A versus $14 \pm 6 \mathrm{mg} / \mathrm{d}$ in group B $(P=\mathrm{NS})$. The mean dose of furosemide was $54 \pm 26 \mathrm{mg} / \mathrm{d}$ in group A versus $55 \pm 28 \mathrm{mg} / \mathrm{d}$ in group B $(P=\mathrm{NS})$. All patients were clinically stable, and no major events occurred during the follow-up period.

\section{Comparison of Changes in Hemodynamics Before and After Treatment}

After $6 \mathrm{mo}$, the systolic and diastolic blood pressure tended to decrease in both groups (group A, $130 \pm 17$ vs. $127 \pm 18 \mathrm{~mm} \mathrm{Hg}$ and $78 \pm 13$ vs. $75 \pm 10 \mathrm{~mm} \mathrm{Hg}$, respectively; group B, $131 \pm 20$ vs. $128 \pm 19 \mathrm{~mm} \mathrm{Hg}$ and $78 \pm 14$ vs. $76 \pm 13 \mathrm{~mm} \mathrm{Hg}$, respectively). However, there were no significant differences in these parameters. Moreover, in both groups, the heart rate did not change significantly after 6 mo (group A, from $72 \pm 12$ to $72 \pm 13 \mathrm{bpm}$; group $\mathrm{B}$, from $74 \pm 14 \mathrm{bpm}$ to $73 \pm 14 \mathrm{bpm}$ ).

\section{Comparison of Cardiac ${ }^{123}$-MIBG Scintigraphic Findings Before and After Treatment}

The $\%$ denervation, $\mathrm{H} / \mathrm{M}$ ratio, and WR data are shown in Table 2. In both groups, the \% denervation decreased significantly after 6 mo compared with the baseline values (group A, $P<0.001$; group $\mathrm{B}, P<0.01$ ). However, the absolute change of $\%$ denervation in group A was significantly greater than that in group B $(P<0.05)$. In both groups, the H/M ratio increased significantly after 6 mo compared with the baseline values (group A, $P<0.001$; group $\mathrm{B}, P<0.01$ ). However, the absolute change of $\mathrm{H} / \mathrm{M}$ ratio in group A was significantly greater than that in group $\mathrm{B}(P<0.05)$. Finally, the WR in both groups decreased significantly after 6 mo compared with the baseline values (group A, $P<0.001$; group $\mathrm{B}, P<0.05$ ). However, the absolute change of WR in group A was significantly greater than that in group B $(P<0.05)$.

Among 3 etiologies (IHD, DCM, and other diseases) at baseline in both groups, ${ }^{123}$ I-MIBG scintigraphic parameters tended to be worse in patients with IHD and tended to be better in patients with other diseases. However, they were not significantly different (Table 3 ). Moreover, the response to therapies was not different in these parameters between baseline and 6 mo after treatment in any disease groups (Table 3).

\section{Comparison of Echocardiographic Findings Before and After Treatment}

The LVEDV, LVESV, and LVEF data are shown in Table 4. In both groups, the LVEDV and LVESV decreased

TABLE 2

Changes in \% Denevation, H/M Ratio, and WR of Patients in Both Groups

\begin{tabular}{|c|c|c|c|c|c|c|}
\hline \multirow[b]{2}{*}{ 123/-MIBG } & \multicolumn{3}{|c|}{ Group A } & \multicolumn{3}{|c|}{ Group B } \\
\hline & Baseline & $6 \mathrm{mo}$ & $\Delta$ & Baseline & $6 \mathrm{mo}$ & $\Delta$ \\
\hline$\%$ denervation & $54 \pm 10$ & $40 \pm 10^{\star}$ & $-14 \pm 12$ & $55 \pm 14$ & $48 \pm 17^{\dagger}$ & $-7 \pm 10^{\ddagger}$ \\
\hline $\mathrm{H} / \mathrm{M}$ ratio & $1.63 \pm 0.23$ & $1.84 \pm 0.19^{\star}$ & $0.19 \pm 0.18$ & $1.68 \pm 0.18$ & $1.76 \pm 0.24^{\dagger}$ & $0.08 \pm 0.14^{\ddagger}$ \\
\hline WR & $47 \pm 10$ & $35 \pm 8^{\star}$ & $-12 \pm 8$ & $46 \pm 10$ & $41 \pm 12^{\S}$ & $-5 \pm 13^{\ddagger}$ \\
\hline \multicolumn{7}{|c|}{$\begin{array}{l}{ }^{\star} P<0.001 \text { vs. baseline. } \\
{ }^{\dagger} P<0.01 \text { vs. baseline. } \\
{ }^{\ddagger} P<0.05 \text { vs. group } A . \\
{ }^{\S} P<0.05 \text { vs. baseline. } \\
\text { Values are mean } \pm S D .\end{array}$} \\
\hline
\end{tabular}


TABLE 3

Changes in \% Denevation, H/M Ratio, and WR of Each Etiology

\begin{tabular}{|c|c|c|c|c|c|c|}
\hline \multirow[b]{3}{*}{ Parameter } & \multicolumn{3}{|c|}{ Group A } & \multicolumn{3}{|c|}{ Group B } \\
\hline & $\mathrm{IHD}$ & DCM & Others & IHD & DCM & Others \\
\hline & $(n=10)$ & $(n=10)$ & $(n=5)$ & $(n=13)$ & $(n=8)$ & $(n=4)$ \\
\hline \multicolumn{7}{|c|}{$\%$ denervation } \\
\hline Baseline & $55 \pm 10$ & $54 \pm 11$ & $53 \pm 11$ & $56 \pm 16$ & $54 \pm 12$ & $54 \pm 13$ \\
\hline $6 \mathrm{mo}$ & $42 \pm 8$ & $40 \pm 12$ & $39 \pm 10$ & $48 \pm 17$ & $47 \pm 18$ & $47 \pm 16$ \\
\hline$\Delta$ & $-13 \pm 7$ & $-14 \pm 13$ & $-14 \pm 11$ & $-8 \pm 10$ & $-7 \pm 10$ & $-7 \pm 11$ \\
\hline \multicolumn{7}{|l|}{ H/M ratio } \\
\hline Baseline & $1.60 \pm 0.27$ & $1.64 \pm 0.19$ & $1.66 \pm 0.23$ & $1.67 \pm 0.24$ & $1.69 \pm 0.18$ & $1.70 \pm 0.17$ \\
\hline $6 \mathrm{mo}$ & $1.80 \pm 0.20$ & $1.84 \pm 0.18$ & $1.85 \pm 0.21$ & $1.75 \pm 0.20$ & $1.76 \pm 0.16$ & $1.78 \pm 0.24$ \\
\hline$\Delta$ & $0.20 \pm 0.19$ & $0.20 \pm 0.18$ & $0.19 \pm 0.22$ & $0.08 \pm 0.20$ & $0.07 \pm 0.13$ & $0.08 \pm 0.14$ \\
\hline \multicolumn{7}{|l|}{ WR } \\
\hline Baseline & $49 \pm 8$ & $46 \pm 14$ & $46 \pm 10$ & $48 \pm 10$ & $46 \pm 12$ & $46 \pm 9$ \\
\hline $6 \mathrm{mo}$ & $36 \pm 11$ & $35 \pm 9$ & $33 \pm 8$ & $43 \pm 14$ & $40 \pm 9$ & $40 \pm 10$ \\
\hline$\Delta$ & $-13 \pm 7$ & $-11 \pm 11$ & $-13 \pm 7$ & $-5 \pm 13$ & $-6 \pm 12$ & $-6 \pm 11$ \\
\hline
\end{tabular}

Values are mean $\pm \mathrm{SD}$.

significantly after 6 mo compared with the baseline values (LVEDV: group A, $P<0.001$, and group $\mathrm{B}, P<0.05$; LVESV: group A, $P<0.001$, and group $\mathrm{B}, P<0.01)$. The LVEF in both groups increased significantly after 6 mo compared with the baseline values (group A, $P<0.001$; group $\mathrm{B}, P<0.05)$. The degree of change in LVEDV, LVESV, and LVEF in group A tended to be more favorable than that in group B, but these changes were not statistically significant.

\section{Comparison of NYHA Functional Class Before and After Treatment}

The NYHA functional class status of the patients is shown in Table 4. Patients from both groups showed improvement after 6 mo of treatment relative to the baseline status (in group A, $P<0.001$; in group $\mathrm{B}, P<0.05$ ). After $6 \mathrm{mo}$, the NYHA functional class status of patients in group A was better than that of patients in group $\mathrm{B}(P<$ $0.05)$.

\section{Comparison of BNP Concentrations Before and After Treatment}

The plasma BNP concentrations are shown in Table 4. In both groups, the plasma BNP concentrations decreased significantly after 6 mo compared with the baseline value (group A, $P<0.001$; group B, $P<0.05$ ). However, the absolute change of BNP in group A was significantly greater than that in group $\mathrm{B}(P<0.05)$.

\section{Relationship Between LV Volume and ${ }^{123}$ I-MIBG Scintigraphic Findings Before and After Treatment}

There were significant correlations between changes in the ${ }^{123} \mathrm{I}-\mathrm{MIBG}$ scintigraphic findings and changes in the LVEDV (\% denervation, $r=0.692, P<0.001$; H/M ratio, $r=-0.437, P<0.05$; and WR, $r=0.505, P<0.01$ ) (Fig. 1 ) or the LDESV in group A (\% denervation, $r=0.663$, $P<0.001 ; \mathrm{H} / \mathrm{M}$ ratio, $r=-0.438, P<0.05$; and WR, $r=$ 0.532, $P<0.01$ ) (Fig. 2). In contrast, there was no

TABLE 4

Changes of LV Volume, LVEF, NHYA Functional Class, and Plasma BNP Concentration of Patients in Both Groups

\begin{tabular}{|c|c|c|c|c|c|c|}
\hline \multirow[b]{2}{*}{ Parameter } & \multicolumn{3}{|c|}{ Group A } & \multicolumn{3}{|c|}{ Group B } \\
\hline & Baseline & $6 \mathrm{mo}$ & $\Delta$ & Baseline & $6 \mathrm{mo}$ & $\Delta$ \\
\hline \multicolumn{7}{|l|}{ Echocardiography } \\
\hline LVEDV (mL) & $192 \pm 32$ & $165 \pm 30^{*}$ & $-27 \pm 26$ & $187 \pm 39$ & $169 \pm 42^{\dagger}$ & $-20 \pm 27$ \\
\hline LVESV (mL) & $131 \pm 30$ & $95 \pm 29^{*}$ & $-36 \pm 26$ & $130 \pm 32$ & $108 \pm 37^{\ddagger}$ & $-22 \pm 28$ \\
\hline LVEF (\%) & $32 \pm 6$ & $43 \pm 11^{*}$ & $11 \pm 10$ & $31 \pm 6$ & $37 \pm 9^{\dagger}$ & $6 \pm 10$ \\
\hline $\mathrm{BNP}(\mathrm{pg} / \mathrm{mL})$ & $249 \pm 133$ & $149 \pm 81^{\star}$ & $-100 \pm 83$ & $243 \pm 119$ & $199 \pm 65^{\dagger}$ & $-43 \pm 97 \S$ \\
\hline NYHA functional class I/II/III/IV & 0/9/16/0 & $8 / 12 / 5 / 0^{\star}$ & & 0/10/15/0 & $3 / 10 / 11 / 1^{\dagger \S}$ & \\
\hline \multicolumn{7}{|l|}{$\begin{array}{l}{ }^{\star} P<0.001 \text { vs. baseline. } \\
{ }^{\dagger} P<0.05 \text { vs. baseline. } \\
{ }^{\ddagger} P<0.01 \text { vs. baseline. } \\
{ }^{\S} P<0.05 \text { vs. group } A .\end{array}$} \\
\hline
\end{tabular}




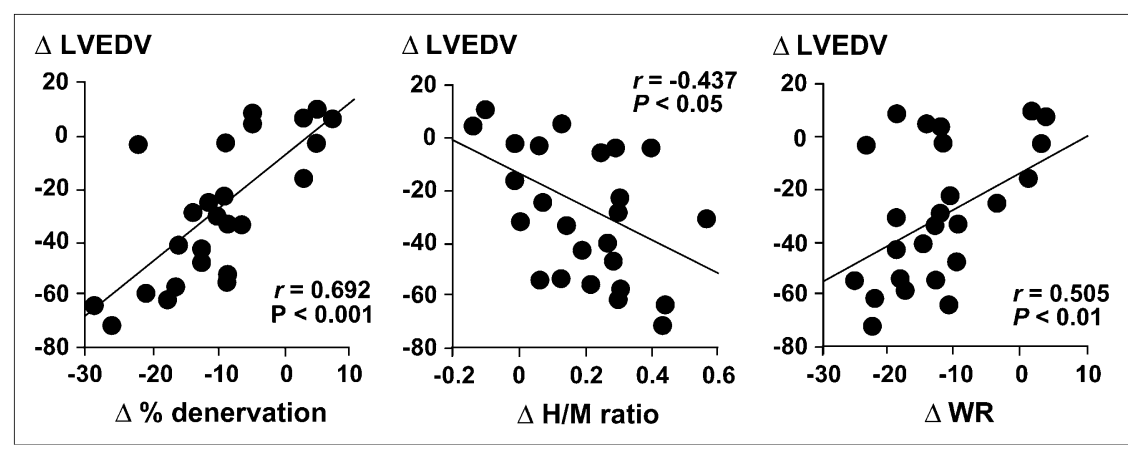

FIGURE 1. Correlations between changes of ${ }^{123}$ I-MIBG scintigraphic findings and LVEDV after combination of spironolactone and candesartan treatment in 25 patients with heart failure. $\Delta$ LVEDV $=$ value of LVEDV after treatment pretreatment value of LVEDV; $\Delta \%$ denervation $=$ value of $\%$ denervation after treatment - pretreatment value of $\%$ denervation; $\Delta \mathrm{H} / \mathrm{M}$ ratio $=$ value of $\mathrm{H} / \mathrm{M}$ ratio after treatment - pretreatment value of $\mathrm{H} / \mathrm{M}$ ratio; $\Delta \mathrm{WR}=$ value of WR after treatment - pretreatment value of WR. relationship between these parameters in group B (Figs. 3 and 4).

\section{DISCUSSION}

Our findings demonstrate that the addition of spironolactone to candesartan can be more beneficial for CSNA and $\mathrm{LV}$ performance than candesartan alone in patients with CHF. Aldosterone promotes retention of sodium and loss of magnesium and potassium and causes myocardial and vascular fibrosis (26), direct vascular damage (27), and baroreceptor dysfunction by altering $\mathrm{Na}, \mathrm{K}-\mathrm{ATPase}$ activity (28). The ARBs target angiotensin production by the RAAS. However, they do not directly block the effects of aldosterone at the receptor level or its deleterious effects on the cardiovascular system (1). Thus, a strategy of providing more complete blockade of aldosterone with the combination of spironolactone and candesartan for the treatment of heart failure appears rational. Naruse et al. (29) reported that the combination of spironolactone and candesartan more strongly inhibits RAAS and has cardioprotective effects compared with candesartan alone in hypertensive rats.

${ }^{123} \mathrm{I}-\mathrm{MIBG}$ is an analog of the adrenergic neuron-blocking agent guanethidine, which is thought to use the same myocardial uptake and release mechanisms as norepinephrine (30). Therefore, cardiac ${ }^{123}$ I-MIBG imaging is a useful tool for detecting abnormalities of the myocardial adrenergic nervous system in patients with $\mathrm{CHF}(5,6)$. Furthermore, many reports have suggested that the treatment of $\mathrm{CHF}$ with ACE inhibitors $(8,9,16)$, ARBs $(13,15,17), \beta$-blockers
$(9,10,19)$, spironolactone $(11,12)$, or torasemide $(18)$ can improve CSNA, on the basis of cardiac ${ }^{123}$ I-MIBG scintigraphic findings. However, to our knowledge, there have been no reports on the additive effects of spironolactone and candesartan on CSNA in patients with CHF compared with candesartan alone. In this study, we examined whether the combination of spironolactone and candesartan improves CSNA in patients with CHF using ${ }^{123} \mathrm{I}-\mathrm{MIBG}$ scintigraphy and found that the \% denervation, $\mathrm{H} / \mathrm{M}$ ratio, and $\mathrm{WR}$ improved with combination therapy compared with candesartan therapy alone.

On the other hand, CSNA evaluated by ${ }^{123}$ I-MIBG scintigraphy also has a useful prognostic value in patients with CHF (6,7). Merlet et al. (6) reported that the delayed $\mathrm{H} / \mathrm{M}$ ratio was the best predictor for survival in $90 \mathrm{CHF}$ patients with reduced cardiac function. Moreover, a multicenter trial from Imamura et al. (7) concluded that the WR was a powerful predictor of subsequent mortality and morbidity in 171 patients with CHF. For these reasons, an increasing effort has been directed toward pharmacologic improvement of CSNA in patients with CHF. This study found that the combination of spironolactone and candesartan treatment significantly improved ${ }^{123}$ I-MIBG scintigraphic parameters compared with candesartan alone. Therefore, further study to compare the long-term effects of combination therapy and candesartan therapy alone on mortality and morbidity in patients with CHF enrolled in this study is warranted.

The plasma BNP concentration is a useful prognostic indicator in patients with $\mathrm{CHF}(31)$, because it is a hormone produced by the left ventricle (32). The plasma BNP

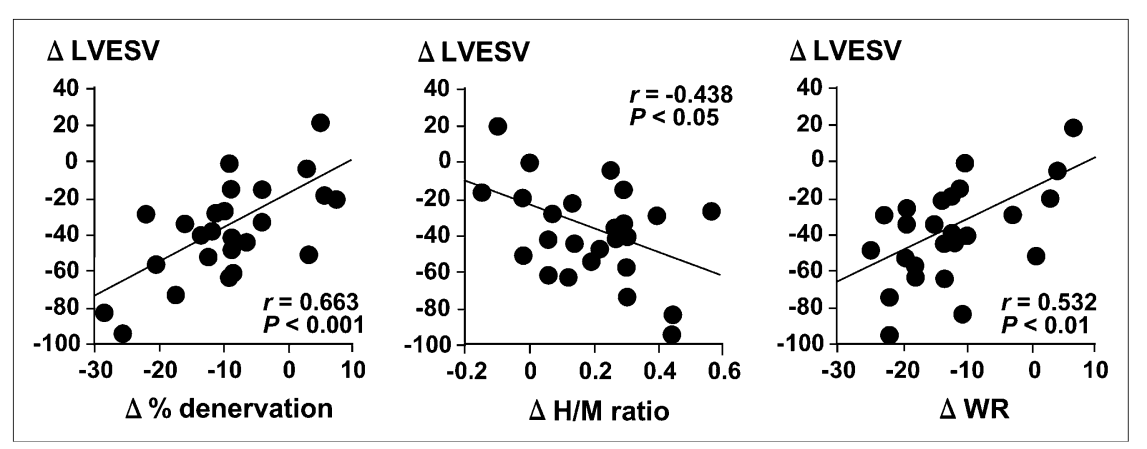

FIGURE 2. Correlations between changes of ${ }^{123}$ I-MIBG scintigraphic findings and LVESV after combination of spironolactone and candesartan treatment in 25 patients with heart failure. $\Delta$ LVESV $=$ value of LVESV after treatment pretreatment value of LVESV; $\Delta \%$ denervation $=$ value of $\%$ denervation after treatment - pretreatment value of \% denervation; $\Delta \mathrm{H} / \mathrm{M}$ ratio $=$ value of $\mathrm{H} / \mathrm{M}$ ratio after treatment - pretreatment value of $\mathrm{H} / \mathrm{M}$ ratio; $\Delta \mathrm{WR}=$ value of $\mathrm{WR}$ after treatment - pretreatment value of WR. 
FIGURE 3. Correlations between changes of ${ }^{123}$ I-MIBG scintigraphic findings and LVEDV after candesartan treatment in 25 patients with heart failure. Abbreviations as in Figure 1.

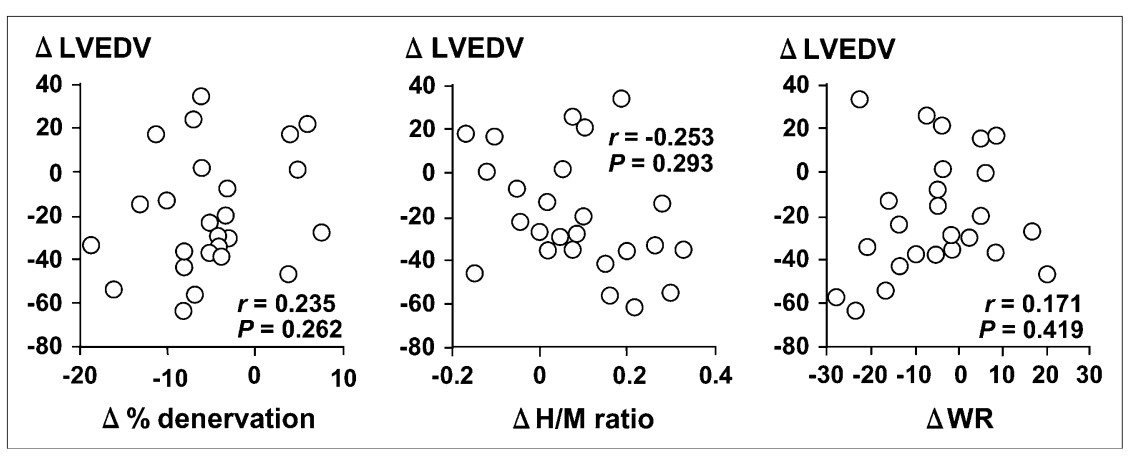

concentration correlates with abnormalities in the LVEF and LV end-diastolic pressure (32) as well as with LV mass (33). Therefore, a decrease in the plasma BNP concentration after combination therapy with spironolactone and candesartan may be due to a decreased LV filling pressure, improvement in LV remodeling, or both factors. A decrease in the BNP concentration during combination therapy may also reflect improvement in LV diastolic function secondary to the effects of this drug on cardiac hypertrophy and fibrosis. Treatment of CHF guided by plasma BNP concentration has been reported to reduce cardiovascular events (31), so a decrease in BNP may be associated with a better outcome, as was the case in previous studies $(4,34)$.

Spironolactone has been reported to improve CSNA $(11,12)$ and decrease myocardial fibrosis in patients with heart failure (35), whereas CSNA and cardiac function are correlated in patients with $\mathrm{CHF}$ (6). In this study, there were significant correlations between changes in the LV volume and the ${ }^{123} \mathrm{I}-\mathrm{MIBG}$ scintigraphic parameters after treatment with a combination of spironolactone and candesartan in patients with CHF. However, no significant correlations were found in the candesartan treatment group. With respect to the influence of spironolactone, it is still unclear whether improvement of LV function (due to the antifibrotic effect of spironolactone) increases myocardial uptake of norepinephrine or whether increased myocardial uptake of norepinephrine leads to improvement in LV function. Therefore, further studies are necessary to clarify the relationship between the improvement of LV function and increased myocardial uptake of norepinephrine. On the other hand, in this study, the relationship between LV volume and \% denervation was more favorable than that between LV volume and H/M ratio or WR. The \% denervation was calculated from SPECT, whereas the H/M ratio and WR were determined from the anterior planar imaging. It is known that lung congestion more strongly affects planar imaging compared with SPECT. All of our study patients had $\mathrm{CHF}$ and reduced cardiac function. Therefore, the baseline data for the H/M ratio and WR might not have been more accurate than that of $\%$ denervation, because baseline ${ }^{123}$ I-MIBG scintigraphy was performed during hospitalization after treatments for acute heart failure. For these reasons, the LV volume might have a better correlation with $\%$ denervation than with $\mathrm{H} / \mathrm{M}$ ratio or $\mathrm{WR}$ in our study.

The relatively small number of patients with $\mathrm{CHF}$ included in our study was one limitation. Despite the group with candesartan therapy alone, the study design omitted the treatment group with a combination of spironolactone and ACE inhibitor as well as the group with ACE inhibitor alone. We need to examine the effects of ${ }^{123} \mathrm{I}-\mathrm{MIBG}$ scintigraphic and echocardiographic parameters using a larger numbers of patients and to compare the effects of the spironolactone plus ACE inhibitor group and ACE inhibitor group with the results of this study.

It has been reported that aldosterone is produced in the ventricles of the failing human heart (36) and that the aldosterone synthase gene is expressed in cardiac tissue (37). The same group has also demonstrated that aldosterone induces the expression of ACE messenger RNA in cultured neonatal cardiocytes (38). Furthermore, Buss et al. (39) reported that aldosterone directly prevents myocardial
FIGURE 4. Correlations between changes of ${ }^{123}$ |-MIBG scintigraphic findings and LVESV after candesartan treatment in 25 patients with heart failure. Abbreviations as in Figure 2.

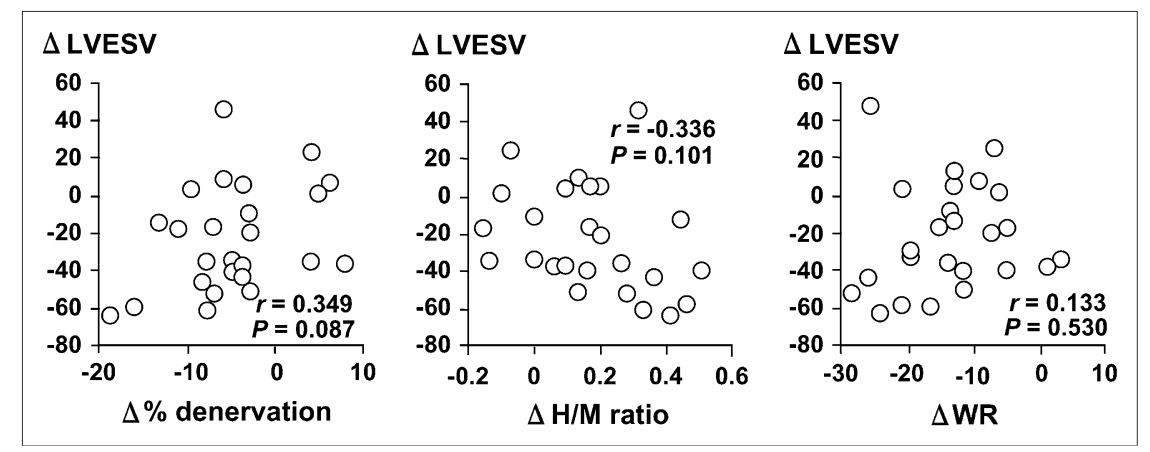


uptake of norepinephrine in an experimental animal model, and the mineralocorticoid receptor-antagonist spironolactone preserves cardiac norepinephrine uptake in the failing heart. We did not measure plasma aldosterone concentrations. However, aldosterone may be produced in cardiac tissue, even if the plasma aldosterone concentration is normal. Therefore, we believe that it is important to inhibit aldosterone produced in cardiac tissue by adding spironolactone to candesartan in patients with $\mathrm{CHF}$.

At our institution (Cardiovascular Hospital of Central Japan), cardiac ${ }^{123}$ I-MIBG scintigraphy is routinely performed using a low-energy, general-purpose collimator. Chen et al. (40) has reported that there is significant septal penetration using this collimator, and septal penetration can degrade image quality and quantitative accuracy. In their study, the optimized protocols based on deconvolution of septal penetration using a low-energy, high-resolution collimator were useful for quantification of ${ }^{123} \mathrm{I}$ cardiac SPECT. Thus, we need to evaluate ${ }^{123}$ I-MIBG SPECT using that protocol in the future.

Currently, many independent reports from different centers around the world support the idea that ${ }^{123}$ I-MIBG myocardial scintigraphy provides useful information for assessing patients with heart failure. However, quantitative ${ }^{123}$ I-MIBG parameters differ between institutions and between instruments, and this tracer is not widely available. For these reasons, cardiac ${ }^{123}$ I-MIBG has yet to achieve broad clinical acceptance, and the evidence supporting the clinical value of this imaging technique remains inadequate. We believe that multicenter studies are needed to establish the efficacy of this imaging modality.

\section{CONCLUSION}

Cardiac ${ }^{123}$ I-MIBG scintigraphic and echocardiographic parameters are improved 6 mo after treatment with both spironolactone and candesartan as well as with candesartan alone. Furthermore, the plasma BNP concentration decreases significantly with both therapies. However, the degree of change in these parameters with combination treatment tends to be better than that with candesartan alone. These findings indicate that the combination of spironolactone and candesartan can be more beneficial for CSNA and LV performance than candesartan alone in patients with CHF.

\section{REFERENCES}

1. McMahon EG. Recent studies with eplerenone, a novel selective aldosterone receptor antagonist. Curr Opin Pharmacol. 2001;1:190-196.

2. Pitt B, Stier CT Jr, Rajagopalan S. Mineralocorticoid receptor blockade: new insights into the mechanism of action in patients with cardiovascular disease. J Renin Angiotensin Aldosterone Syst. 2003;4:164-168.

3. Stier CT Jr, Chander PN, Rocha R. Aldosterone as a mediator in cardiovascular injury. Cardiol Rev. 2002;10:97-107.

4. Pfeffer MA, Swedberg K, Granger CB, et al. CHARM Investigators and Committees: effects of candesartan on mortality and morbidity in patients with chronic heart failure-the CHARM-overall programme. Lancet. 2003;362:759-766.

5. Schofer J, Spielmann R, Schuchert A, Weber K, Schluter M. Iodine-123 metaiodobenzylguanidine scintigraphy: a noninvasive method to demonstrate myo- cardial adrenergic nervous system disintegrity in patients with idiopathic dilated cardiomyopathy. J Am Coll Cardiol. 1988;12:1252-1258.

6. Merlet P, Valette H, Dubois-Rande JL, et al. Prognostic value of cardiac metaiodobenzylguanidine imaging in patients with heart failure. J Nucl Med. 1992;33:471-477.

7. Imamura Y, Fukuyama T, Mochizuki T, Miyagawa M, Watanabe K: Ehime MIBG Heart Failure Study Investigators. Prognostic value of iodine-123-metaiodobenzylguanidine imaging and cardiac natriuretic peptide levels in patients with left ventricular dysfunction resulting from cardiomyopathy. Jpn Circ J. 2001;65:155-160.

8. Takeishi Y, Atsumi H, Fujiwara S, Takahashi K, Tomoike H. ACE inhibition reduces cardiac iodine-123-MIBG release in heart failure. J Nucl Med. 1997;38: 1085-1089.

9. Toyama T, Aihara Y, Iwasaki T, et al. Cardiac sympathetic activity estimated by ${ }^{123}$ I-MIBG myocardial imaging in patients with dilated cardiomyopathy after beta-blocker or angiotensin-converting enzyme inhibitor therapy. J Nucl Med. 1999;40:217-223.

10. Toyama T, Hoshizaki H, Seki R, et al. Efficacy of carvedilol treatment on cardiac function and cardiac sympathetic nerve activity in patients with dilated cardiomyopathy: comparison with metoprolol therapy. J Nucl Med. 2003;44:1604-1611.

11. Kasama S, Toyama T, Kumakura H, et al. Spironolactone improves cardiac sympathetic nerve activity and symptoms in patients with congestive heart failure. J Nucl Med. 2002;43:1279-1285.

12. Kasama S, Toyama T, Kumakura H, et al. Effect of spironolactone on cardiac sympathetic nerve activity and left ventricular remodeling in patients with dilated cardiomyopathy. J Am Coll Cardiol. 2003;41:574-581.

13. Kasama S, Toyama $\mathrm{T}$, Kumakura $\mathrm{H}$, et al. Addition of valsartan to an angiotensin-converting enzyme inhibitor improves cardiac sympathetic nerve activity and left ventricular function in patients with congestive heart failure. J Nucl Med. 2003;44:884-890.

14. Kasama S, Toyama T, Kumakura H, et al. Effects of intravenous atrial natriuretic peptide on cardiac sympathetic nerve activity in patients with decompensated congestive heart failure. J Nucl Med. 2004;45:1108-1113.

15. Kasama S, Toyama T, Kumakura H, et al. Effects of candesartan on cardiac sympathetic nerve activity in patients with congestive heart failure and preserved left ventricular ejection fraction. J Am Coll Cardiol. 2005;45:661-667.

16. Kasama S, Toyama T, Kumakura H, et al. Effects of perindopril on cardiac sympathetic nerve activity in patients with congestive heart failure: comparison with enalapril. Eur J Nucl Med Mol Imaging. 2005;32:964-971.

17. Kasama S, Toyama T, Hatori T, et al. Comparative effects of valsartan with enalapril on cardiac sympathetic nerve activity and plasma brain natriuretic peptide in patients with congestive heart failure. Heart. 2006;92:625-630.

18. Kasama S, Toyama $\mathrm{T}$, Hatori $\mathrm{T}$, et al. Effects of torasemide on cardiac sympathetic nerve activity and left ventricular remodelling in patients with congestive heart failure. Heart. 2006;92:1434-1440.

19. Kasama S, Toyama T, Hatori T, et al. Evaluation of cardiac sympathetic nerve activity and left ventricular remodelling in patients with dilated cardiomyopathy on the treatment containing carvedilol. Eur Heart J. 2007;28:989-995.

20. Schiller NB, Shah PM, Crawford M, et al. Recommendations for quantitation of the left ventricle by two-dimensional echocardiography: American Society of Echocardiography Committee on Standards-Subcommittee on Quantitation of Two-Dimensional Echocardiograms. J Am Soc Echocardiogr. 1989;2: $358-367$.

21. Kasama S, Toyama T, Kumakura H, et al. Effects of nicorandil on cardiac sympathetic nerve activity after reperfusion therapy in patients with first anterior acute myocardial infarction. Eur J Nucl Med Mol Imaging. 2005;32:322-328.

22. Kasama S, Toyama $\mathrm{T}$, Hatori $\mathrm{T}$, et al. Effects of intravenous atrial natriuretic peptide on cardiac sympathetic nerve activity and left ventricular remodeling in patients with first anterior acute myocardial infarction. J Am Coll Cardiol. 2007; 49:667-674.

23. Kasama S, Toyama T, Sumino H, et al. Long-term nicorandil therapy improves cardiac sympathetic nerve activity after reperfusion therapy in patients with first acute myocardial infarction. J Nucl Med. 2007;48:1676-1682.

24. Cerqueira MD, Weissman NJ, Dilsizian V, et al. American Heart Association Writing Group on Myocardial Segmentation and Registration for Cardiac Imaging: standardized myocardial segmentation and nomenclature for tomographic imaging of the heart-a statement for healthcare professionals from the Cardiac Imaging Committee of the Council on Clinical Cardiology of the American Heart Association. Circulation. 2002;105:539-542.

25. Kasama S, Toyama T, Kumakura H, et al. Dobutamine stress ${ }^{99 \mathrm{~m}} \mathrm{Tc}$-tetrofosmin quantitative gated SPECT predicts improvement of cardiac function after carvedilol treatment in patients with dilated cardiomyopathy. J Nucl Med. 2004;45:18781884 .

26. Weber KT, Brilla CG. Pathological hypertrophy and cardiac interstitium: fibrosis and renin-angiotensin-aldosterone system. Circulation. 1991;83:1849-1865. 
27. Rocha R, Chander PN, Khanna K, Zuckerman A, Stier CT Jr. Mineralocorticoid blockade reduces vascular injury in stroke-prone hypertensive rats. Hypertension. 1998;31:451-458.

28. Wang W. Chronic administration of aldosterone depresses baroreceptor reflex function in the dog. Hypertension. 1994;24:571-575.

29. Naruse M, Tanabe A, Sato A, et al. Aldosterone breakthrough during angiotensin II receptor antagonist therapy in stroke-prone spontaneously hypertensive rats. Hypertension. 2002;40:28-33.

30. Wieland DM, Wu J, Brown LE, Mangner TJ, Swanson DP, Beierwaltes WH. Radiolabeled adrenergic neuron-blocking agents: adrenomedullary imaging with [131 I] iodobenzylguanidine. J Nucl Med. 1980;21:349-353.

31. Tsutamoto $\mathrm{T}$, Wada A, Maeda $\mathrm{K}$, et al. Attenuation of compensation of endogenous cardiac natriuretic peptide system in chronic heart failure: prognostic role of plasma brain natriuretic peptide concentration in patients with chronic symptomatic left ventricular dysfunction. Circulation. 1997;96: 509-516.

32. Yasue H, Yoshimura M, Sumida $H$, et al. Localization and mechanism of secretion of B-type natriuretic peptide in comparison with those of A-type natriuretic peptide in normal subjects and patients with heart failure. Circulation. 1994;90:195-203.

33. Kohno M, Horio T, Yokokawa K, et al. Brain natriuretic peptide as a cardiac hormone in essential hypertension. Am J Med. 1992;92:29-34.
34. Pitt B, Zannad F, Remme WJ, et al. The effect of spironolactone on morbidity and mortality in patients with severe heart failure: Randomized Aldactone Evaluation Study Investigators. N Engl J Med. 1999;341:709-717.

35. Zannad F, Alla F, Dousset B, Perez A, Pitt B. Limitation of excessive extracellular matrix turnover may contribute to survival benefit of spironolactone therapy in patients with congestive heart failure: insights from the Randomized Aldactone Evaluation Study (RALES)—Rales Investigators. Circulation. 2000; 102:2700-2706.

36. Mizuno Y, Yoshimura M, Yasue H, et al. Aldosterone production is activated in failing ventricle in humans. Circulation. 2001;103:72-77.

37. Yoshimura M, Nakamura S, Ito T, et al. Expression of aldosterone synthase gene in failing human heart: quantitative analysis using modified real-time polymerase chain reaction. J Clin Endocrinol Metab. 2002;87:3936-3940.

38. Harada E, Yoshimura M, Yasue $\mathrm{H}$, et al. Aldosterone induces angiotensinconverting-enzyme gene expression in cultured neonatal rat cardiocytes. Circulation. 2001;104:137-139.

39. Buss SJ, Backs J, Kreusser MM, et al. Spironolactone preserves cardiac norepinephrine reuptake in salt-sensitive Dahl rats. Endocrinology. 2006;147: 2526-2534.

40. Chen J, Garcia EV, Galt JR, Folks RD, Carrio I. Optimized acquisition and processing protocols for I-123 cardiac SPECT imaging. J Nucl Cardiol. 2006; 13:251-260. 